\title{
Nurse Staffing and Health Outcomes of Psychiatric Inpatients: A Secondary Analysis of National Health Insurance Claims Data
}

\author{
Park, Suin ${ }^{1 \oplus} \cdot$ Park, Sohee $^{2 \oplus} \cdot$ Lee, Young Joo ${ }^{3,4 \oplus} \cdot$ Park, Choon-Seon $^{5 \oplus} \cdot$ Jung, Young-Chu $^{6,7 \odot} \cdot$ Kim, Sunah $^{8,9 \circledast}$ \\ ${ }^{1}$ College of Nursing, Kosin University, Busan \\ ${ }^{2}$ Department of Biostatistics, Graduate School of Public Health, Yonsei University, Seoul \\ ${ }^{3}$ College of Nursing, Daegu Catholic University, Daegu \\ ${ }^{4}$ Research Institute of Nursing Science, Daegu Catholic University, Daegu \\ ${ }^{5}$ Department of Quality Assessment Administration, Health Insurance Review and Assessment Service, Wonju \\ ${ }^{6}$ Department of Psychiatry, College of Medicine, Yonsei University, Seoul \\ ${ }^{7}$ Institute of Behavioral Science in Medicine, College of Medicine, Yonsei University, Seoul \\ ${ }^{8}$ College of Nursing, Yonsei University, Seoul \\ ${ }^{9}$ Mo-Im Kim Nursing Research Institute, College of Nursing, Yonsei University, Seoul, Korea
}

\begin{abstract}
Purpose: The present study investigated the association between nurse staffing and health outcomes among psychiatric inpatients in Korea by assessing National Health Insurance claims data. Methods: The dataset included 70,136 patients aged 19 years who were inpatients in psychiatric wards for at least two days in 2016 and treated for mental and behavioral disorders due to use of alcohol; schizophrenia, schizotypal and delusional disorders; and mood disorders across 453 hospitals. Nurse staffing levels were measured in three ways: registered nurse-to-inpatient ratio, registered nurse-to-adjusted inpatient ratio, and nursing staff-to-adjusted inpatient ratio. Patient outcomes included length of stay, readmission within 30 days, psychiatric emergency treatment, use of injected psycholeptics for chemical restraint, and hypnotics use. Relationships between nurse staffing levels and patient outcomes were analyzed considering both patient and system characteristics using multilevel modeling. Results: Multilevel analyses revealed that more inpatients per registered nurse, adjusted inpatients per registered nurse, and adjusted inpatients per nursing staff were associated with longer lengths of stay as well as a higher risk of readmission. More adjusted inpatients per registered nurse and adjusted inpatients per nursing staff were also associated with increased hypnotics use but a lower risk of psychiatric emergency treatment. Nurse staffing levels were not significantly associated with the use of injected psycholeptics for chemical restraint. Conclusion: Lower nurse staffing levels are associated with negative health outcomes of psychiatric inpatients. Policies for improving nurse staffing toward an optimal level should be enacted to facilitate better outcomes for psychiatric inpatients in Korea.
\end{abstract}

Key words: Mental Disorders; Patient Outcome Assessment; Psychiatric Nursing

\footnotetext{
* 이 논문은 제 1 저자 박수인의 박사학위논문의 축약본임.

* 본 연구는 2019년도 연세대학교 간호대학 김모임간호학연구소의 지원 및 연세대학교 간호대학 이연순장학금 지원에 의해 수행되었음.

* 이 논문은 2019년 12th International Nursing Conference에서 발표되었음.

* This manuscript is a condensed form of the first author's doctoral dissertation from Yonsei University. Year of 2019.

* This study was supported by the Yonsei University College of Nursing Mo-Im Kim Nursing Research Institute, and 'Yeonsun Lee Scholarship' at Yonsei University College of Nursing.

* This work was presented at 12th International Nursing Conference, October, 2019, Seoul, Korea.
}

Address reprint requests to : Kim, Sunah

College of Nursing, Yonsei University, 50-1 Yonsei-ro, Seodaemun-gu, Seoul 03722, Korea

Tel: + 82-2-2228-3275 Fax: +82-2-392-5440 E-mail: psy0962@yuhs. ac

Received: October 1, 2019 Revised: March 30, 2020 Accepted: March 31, 2020 Published online June 30, 2020

This is an Open Access article distributed under the terms of the Creative Commons Attribution NoDerivs License. (http://creativecommons. org/licenses/by-nd/4. 0)

If the original work is properly cited and retained without any modification or reproduction, it can be used and re-distributed in any format and medium. 


\section{INTRODUCTION}

Although Korea has observed substantial improvements to the quality of mental health care services, national statistics suggest further efforts are needed to improve mental health care efficacy. The average length of stay (LOS) for psychiatric patients has increased in Korea over the last 10 years [1]. For instance, the average LOS in 2017 was 168 days, five times higher than the average observed within other Organization for Economic Cooperation and Development (OECD) countries (29.4 days). In 2011, the psychiatric readmission rate for schizophrenia in Korea was 19.4\%, the second highest among all OECD countries [2]. Additionally, incidence rates of psychiatric emergency treatment and the use of hypnotics among patients (diagnosed with mental and behavioral disorders due to use of alcohol; schizophrenia, schizotypal and delusional disorders; and mood disorders) showed an increasing trend between 2015 to 2019 in Korea [3,4]. Psychiatric emergency treatment (reimbursed by Korean National Health Insurance) includes assessing patients' psychiatric symptoms through emergent interviews, applying seclusion and restraints in order to prevent harmful behaviors to themselves or others, and ongoing assessments until stabilization [5]. Thus, investigating factors associated with LOS, readmissions, and treatment amount, including pharmacological treatment, is worthwhile.

Nurse staffing is an important factor that influences patient safety and recovery. Staffing issues are also important for health policy provisions that establish domestic legal standards, which ensure compliance at each hospital. In Korea, standards for nurse staffing in psychiatric wards are stipulated in the Enforcement Rule of the Act on the Improvement of Mental Health and the Support for Welfare Services for Mental Patients, Article 11, Paragraph 2 [6]. According to this Article, one nurse is assigned to 13 psychiatric inpatients, and half of the registered nurse capacity can be substituted by nurse assistants [6]. This standard is considered insufficient to ensure adequate nurse staffing in comparison with standards elsewhere. For example, in the US state of California licensed nurse-to-patient ratio during one shift for acute psychiatric wards is 1:6 [7], while in Japan the employed nurse-to-inpatient ratios for the number of psychiatric beds are $1: 3$ or $1: 4[8,9]$. Therefore, it is essential to identify associations between nurse staffing and health outcomes among psychiatric inpatients in order to review and revise legal standards in Korea. According to previous studies, higher nurse staffing levels were linked to reduced LOS $[10,11]$, readmission rates $[12,13]$, and mortality among medical or surgical patients [14]. Higher nurse staffing levels were also associated with fewer medical error incidences, pressure ulcers, and patient falls with injuries [15]. Several studies also examined associations between nurse staffing and health outcomes among psychiatric inpatients. Some reported that a greater number of psychiatric nurses were related to decreased LOS $[16,17]$, and more total nurses were associated with fewer psychiatric readmissions within 30 days [18]. Other studies investigated the relationship between nurse staffing and seclusion/restraint use, observing inconsistent results [19-22]. However, the amount of research conducted in this area is limited, especially in Korea. Moreover, the research conducted to date focuses on the influence of nurse staffing on general health outcomes among psychiatric inpatients considering a few health outcome variables. Therefore, this issue warrants further attention.

Health outcomes related to psychiatric nursing care services can include LOS, readmissions, use of seclusion/restraints, use of injected psycholeptics for chemical restraint, and hypnotics use. Improving the quality of care services with adequate nurse staffing was previously shown to help prevent unnecessary extension of hospitalizations and readmissions [23,24]. Research suggested that mental health nurses played an important role in reducing restrictive interventions, such as seclusion and mechanical or chemical restraint, by providing therapeutic interventions to aid patients who are at risk of harming themselves or others $[25,26]$. Furthermore, sleep disturbances were prevalent among psychiatric patients [27], and nurses were in a prime position to help patients achieve adequate sleep by providing environmental, non-pharmacological, and pharmacological interventions [28,29]. Thus, it is necessary to investigate the relationship between nurse staffing and these important patient outcomes within psychiatric wards. The present study investigated the association between nurse staffing and health outcomes of psychiatric inpatients (LOS, readmission, psychiatric emergency treatment, use of injected psycholeptics, and hypnotics use) using National Health Insurance claims data from Korea. 


\section{Conceptual framework}

The conceptual framework for this study was based on the Patient Care Delivery Model (PCDM) which conceptualizes the delivery of nursing care services to a patient care unit within a hospital [30]. It focused on the interdependent and dynamic interactions among inputs (characteristics of patients, nurses, systems, and system behaviors), throughputs (interventions, perceived work environment, and environmental complexity), intermediate outputs (staffing utilization level), and distal outputs (patient, nurse, and system outputs). PCDM proposed that the inputs yield distal outputs through the throughput processes which are influenced by throughput factors in the patient care delivery subsystem and affect the intermediate outputs. This study focused on the relationship between input factors, especially system behaviors such as nurse staffing levels, and output factors which are finally obtained from input factors as the intermediate variables that could be included in the throughput processes but were not available in the National Health Insurance claims data. Input factors consist of patient and system characteristics as well as system behaviors, such as nurse staffing level. Output factors consist of LOS, readmission within 30 days, psychiatric emergency treatment, use of injected psycholeptics for chemical restraint, and hypnotics use (Supplement 1).

\section{METHODS}

\section{Design}

This study was a secondary analysis of National Health Insurance claims data aiming to examine the relationships between nurse staffing level and health outcomes of psychiatric inpatients.

\section{Data source}

We used the National Health Insurance claims data submitted to the Health Insurance Review and Assessment Service (HIRA). Data were submitted by psychiatric wards from tertiary and general hospitals, hospitals (see below), psychiatric hospitals, and clinics. In Korea, based on the Enforcement Rule of the Act on the Improvement of Mental Health and the Support for Welfare Services for Mental Patients, psychiatric clinics were allowed to have 49 or fewer beds for inpatient care. One hospital type in
Korea (designated as simply "hospitals" above) may be regarded as an intermediate stage between that of clinics and general hospitals in terms of the number of departments, beds, facilities, or a staffing capacity. We also used medical resource information data and estimation reports on differentiating policies for hospitalization fees in medical aid psychiatric departments provided by the HIRA. After obtaining approval from the Institutional Review Board of the Y University Health System (Approval No.: Y-20180089) and approval for our research project from the HIRA, we were provided with HIRA data. The dataset did not include any information on the individuals or hospitals and did not allow for data export. To construct a dataset for the analyses in the current study, we selected all claims data submitted to the HIRA for psychiatric inpatient care in 2016 and combined it with hospital data (medical resource information data and estimation reports on differentiating policies for hospitalization fees in medical aid psychiatric departments) from the same year. As this study was a secondary analysis of public access data, it did not require informed consent.

\section{Study sample}

The final data comprised 70,136 inpatients from 453 hospitals after screening for inclusion and exclusion criteria. Figure 1 showed the process of the data construction. When merging patient data and hospital data, 5,434 patients had no corresponding hospital data and so were removed. The final dataset had no missing components.

\section{1) Inclusion criteria}

We included the data from tertiary hospitals, general hospitals, hospitals, psychiatric hospitals, and clinics that 1) claimed reimbursement for psychiatric inpatient services whose primary diagnosis code was F10.x, F2.x, or F3.x according to the International Statistical Classification of Diseases and Related Health Problems, $10^{\text {th }}$ Revision (ICD-10); 2) submitted estimation reports on the differentiating policies for hospitalization fees in medical aid psychiatric departments; 3) reported at least one registered nurse $(\mathrm{RN})$ and three or more nursing staff working for psychiatric departments; and 4) treated at least one inpatient per day in a psychiatric ward. According to ICD-10, F10-code indicates "mental and behavioral disorders due to use of alcohol"; the F2- 
Hospital data

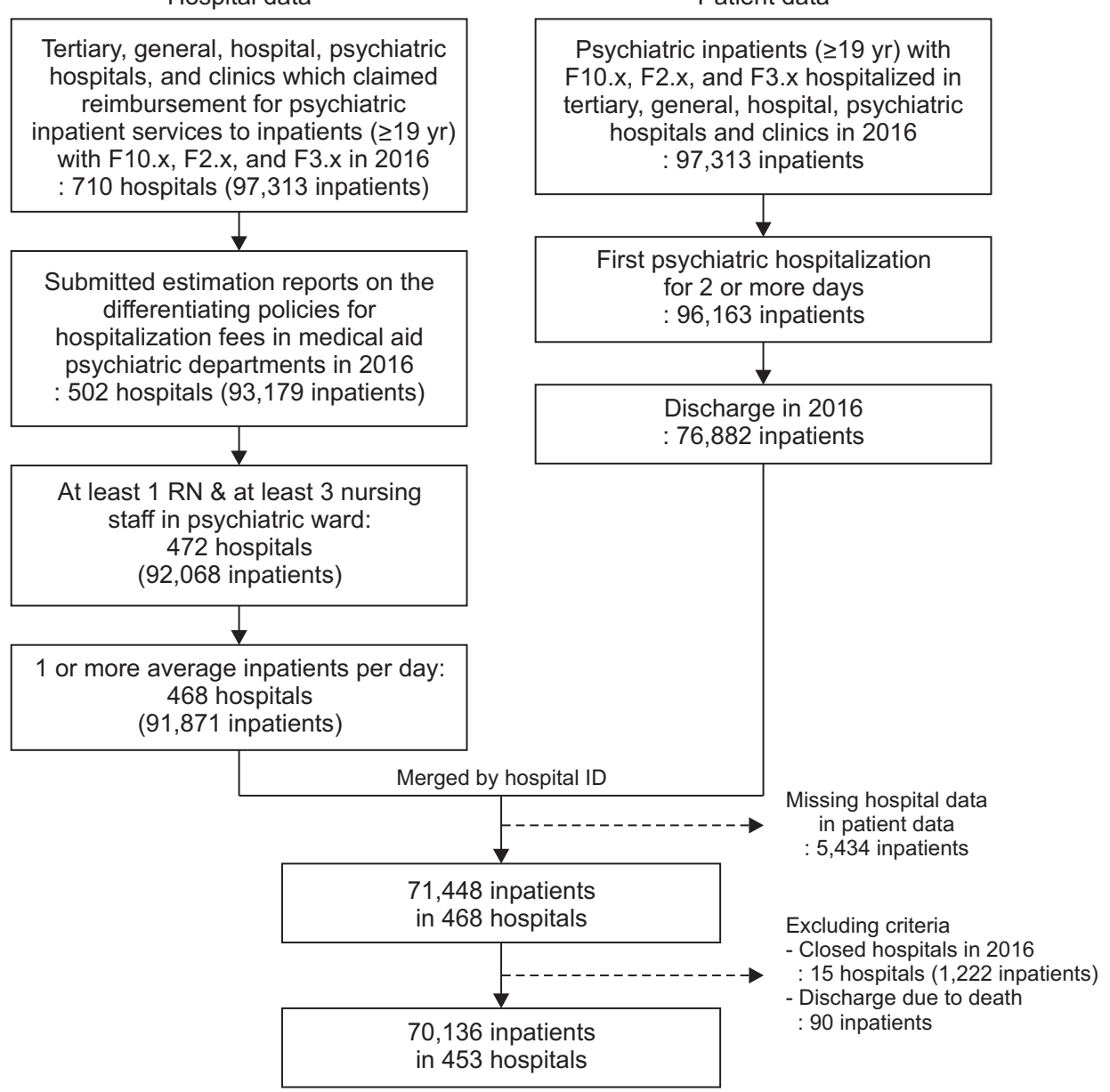

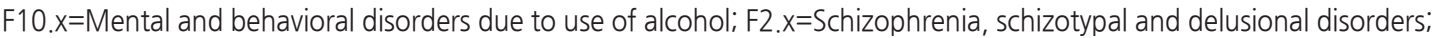
F3. $x=$ Mood disorders; Nursing staff=Total of registered nurses and nurse assistants; RN=Registered nurse.

Figure 1. Process of data construction (Park Suin. Nurse staffing and health outcomes of psychiatric inpatients [dissertation]. 2019. p.22).

code indicates "schizophrenia, schizotypal or delusional disorders"; and F3-code indicates "mood disorders". These diagnostic groups were selected considering specific clinical manifestations such as psychiatric symptoms and treatment mechanisms $[18,27,31]$.

We included data from patients aged 19 years or older who stayed in a psychiatric ward for at least two days in 2016. All patients had F10.x, F2.x, or F3.x as the primary diagnosis. Patients who stayed for two or more days in 2016 needed to be discharged the same year in order to be included. If patients were hospitalized several times in 2016, the first hospitalization was selected for analysis.

\section{2) Exclusion criteria}

Hospitals that closed in 2016 were excluded, and patients who were discharged due to death were excluded.

\section{Nurse staffing level}

Nurse staffing level refers to the nurse-to-patient ratio (number of patients per nurse) and was measured in three ways.

\section{1) RN-to-inpatient ratio}

The RN-to-inpatient ratio (number of inpatients per $\mathrm{RN}$ ) was calculated as the average number of inpatients per day divided by the average number of employed psychiatric nurses per day in each hospital. 


\section{2) RN-to-adjusted inpatient ratio}

The RN-to-adjusted inpatient ratio (number of adjusted inpatients per $\mathrm{RN}$ ) was calculated as the average number of adjusted inpatients per day divided by the average number of employed psychiatric nurses per day in each hospital. The number of adjusted inpatients was the sum of the inpatients, outpatients, and daycare patients. This considered three outpatients as one inpatient, and two daycare patients as one inpatient, based on the Enforcement Rule of the Act on the Improvement of Mental Health and the Support for Welfare Services for Mental Patients, Article 11, Paragraph 2 [6].

\section{3) Nursing staff-to-adjusted inpatient ratio}

The nursing staff-to-adjusted inpatient ratio (number of adjusted inpatients per nursing staff) was measured as the average number of adjusted inpatients per day divided by the average number of employed psychiatric nursing staff per day in each hospital. The nursing staff is the sum of the total number of RNs and nurse assistants working in the inpatient ward, outpatient department, and daycare ward within the mental health department. The number of nurse assistants was counted as nursing staff within the range of half of the total number of nursing staff [6].

Each nurse staffing variable was included separately in the multilevel models: Model A included the RN-to-inpatient ratio; Model B included the RN-to-adjusted inpatient; and Model C included the nursing staff-to-adjusted inpatient ratio.

\section{Health Outcomes}

Dependent variables in this study included five outcomes measured at the patient level.

\section{1) Length of stay (LOS)}

LOS was calculated as the total number of days in psychiatric hospitalization due to F10.x, F2.x, or F3.x as the primary diagnosis.

\section{2) Readmission within 30 days}

We calculated psychiatric readmission within 30 days after discharge, including readmissions to the same ward and other psychiatric ward. We excluded cases in which the discharge and readmission dates were the same, as well as patients who were discharged from December 2 to 31, 2016, as we were unavailable to fully trace readmission within 30 days post-discharge.

\section{3) Psychiatric emergency treatment}

As mentioned above, psychiatric emergency treatment, coded NN100 in the National Health Insurance claims data, is defined as assessing patients' psychiatric symptoms through emergent interviews followed by the administration of seclusion and restraints to prevent harmful behaviors to themselves or others [5]. Psychiatric emergency treatment was calculated as the total number of NN100 code claims filed during an inpatient's hospitalization.

\section{4) Use of injected psycholeptics for chemical restraint}

Use of injected psycholeptics for chemical restraint refers to the cumulative Defined Daily Dose (DDD) of haloperidol, lorazepam, and/or olanzapine in parenteral injections classified as tranquilizers (Classification Code 117) by the Ministry of Food and Drug Safety based on the Regulation on Codes for Classification of Drugs and Other Products [32,33], with the exception of long-acting agents.

\section{5) Hypnotics use}

Hypnotics use was the DDD of flunitrazepam, flurazepam, triazolam, and/or zolpidem (benzodiazepine derivatives and benzodiazepine-related drugs group), as well as doxepin (the antidepressants group) based on the Anatomical Therapeutic Chemical classification [32] among oral agents classified as hypnotics and sedatives (Classification Code 112) by the Ministry of Food and Drug Safety based on the Regulation on Codes for Classification of Drugs and Other Products [33].

\section{Patient and system characteristics}

We adjusted our multilevel analyses for both patient and system characteristics (selected from within the dataset and based on previous studies) to examine the associations between nurse staffing levels and patient outcomes. Patient characteristics included age, gender, type of insurance, diagnosis, previous psychiatric hospitalization within the last year, number of psychiatric sub-diagnoses, number of physical sub-diagnoses, and Elix- 
hauser Comorbidity Measures (ECM) score for the last year [3437]. LOS, which is one of the outcomes, was additionally adjusted as a patient characteristic when analyzing the association between nurse staffing levels and other outcomes (readmission, psychiatric emergency treatments, use of injected psycholeptics for chemical restraint, and hypnotics use) based on previous studies $[18,21,38]$. An insomnia sub-diagnosis was also additionally ad- justed during the analysis of the relationship between nurse staffing and hypnotics use; this is because hypnotic drugs are usually prescribed to treat insomnia [39]. System characteristics included type of hospital, size, ownership, teaching, location, bed operation rate, and RN proportion (the ratio of RNs to total nursing staff) $[17,18,34,37,40]$. We did not adjust for the number of doctors per 100 patients due to the number of doctors being

Table 1. Patient and System Characteristics, System Behaviors, and Health Outcomes at the Individual Level

$(N=70,136)$

\begin{tabular}{|c|c|c|c|c|c|c|c|c|c|c|c|}
\hline \multirow[t]{2}{*}{ Variables } & \multirow[t]{2}{*}{$\mathrm{n}(\%)$} & \multicolumn{2}{|c|}{ Length of stay } & \multicolumn{2}{|c|}{$\begin{array}{c}\text { Readmission } \\
\text { within } 30 \text { days } \\
(n=65,433)^{+}\end{array}$} & \multicolumn{2}{|c|}{$\begin{array}{c}\text { Psychiatric emergency } \\
\text { treatment }\end{array}$} & \multicolumn{2}{|c|}{$\begin{array}{l}\text { Use of injected } \\
\text { psycholeptics }\end{array}$} & \multicolumn{2}{|c|}{ Hypnotics use } \\
\hline & & $\mathrm{M} \pm \mathrm{SD}$ & $p$ & $\%$ & $p$ & $\mathrm{M} \pm \mathrm{SD}$ & $p$ & $\mathrm{M} \pm \mathrm{SD}$ & $p$ & $\mathrm{M} \pm \mathrm{SD}$ & $p$ \\
\hline \multicolumn{12}{|l|}{ Patient characteristics } \\
\hline \multicolumn{12}{|l|}{ Age group (yr) } \\
\hline$<40^{\mathrm{a}}$ & $15,721(22.4)$ & $42.48 \pm 48.31$ & $<.001$ & 20.2 & $<.001$ & $0.28 \pm 0.58$ & $<.001$ & $3.50 \pm 9.11$ & $<.001$ & $0.25 \pm 0.48$ & $<.001$ \\
\hline $40 \sim 49^{b}$ & $17,129(24.4)$ & $50.81 \pm 56.19$ & $c>b, d>a$ & 25.4 & & $0.25 \pm 0.54$ & $a>b>c>d$ & $2.75 \pm 6.75$ & $a>b>c>d$ & $0.36 \pm 0.59$ & $b, c>d>a$ \\
\hline $50 \sim 59 c$ & $20,192(28.8)$ & $52.90 \pm 58.59$ & & 26.3 & & $0.23 \pm 0.50$ & & $2.28 \pm 5.30$ & & $0.37 \pm 0.63$ & \\
\hline$\geq 60^{d}$ & $17,094(24.4)$ & $51.25 \pm 57.45$ & & 18.2 & & $0.20 \pm 0.48$ & & $1.78 \pm 5.10$ & & $0.28 \pm 0.50$ & \\
\hline \multicolumn{12}{|l|}{ Gender } \\
\hline Male & $44,105(62.9)$ & $52.16 \pm 57.69$ & $<.001$ & 25.3 & $<.001$ & $0.25 \pm 0.54$ & $<.001$ & $2.49 \pm 6.43$ & .003 & $0.34 \pm 0.57$ & $<.001$ \\
\hline Female & $26,031(37.1)$ & $45.40 \pm 51.86$ & & 18.3 & & $0.21 \pm 0.50$ & & $2.64 \pm 7.06$ & & $0.26 \pm 0.51$ & \\
\hline \multicolumn{12}{|l|}{ Type of insurance } \\
\hline Medical aid & 27,394 (39.1) & $61.01 \pm 63.39$ & $<.001$ & 31.6 & $<.001$ & $0.18 \pm 0.50$ & $<.001$ & $2.17 \pm 6.17$ & $<.001$ & $0.38 \pm 0.61$ & $<.001$ \\
\hline Health insurance & $42,742(60.9)$ & $42.37 \pm 48.78$ & & 17.0 & & $0.27 \pm 0.54$ & & $2.78 \pm 6.96$ & & $0.27 \pm 0.50$ & \\
\hline \multicolumn{12}{|l|}{ Diagnosis } \\
\hline F10. $x^{a}$ & $26,498(37.8)$ & $50.07 \pm 55.82$ & $<.001$ & 27.0 & $<.001$ & $0.29 \pm 0.55$ & $<.001$ & $2.37 \pm 4.86$ & $<.001$ & $0.40 \pm 0.58$ & $<.001$ \\
\hline$F 2 . x^{b}$ & $24,267(34.6)$ & $60.19 \pm 61.20$ & $b>a>c$ & 23.4 & & $0.21 \pm 0.54$ & $a>b>c$ & $2.58 \pm 7.72$ & $b, c>a$ & $0.21 \pm 0.45$ & $a>c>b$ \\
\hline$F 3 . x^{c}$ & $19,371(27.6)$ & $35.89 \pm 44.14$ & & 15.9 & & $0.19 \pm 0.47$ & & $2.73 \pm 7.35$ & & $0.34 \pm 0.58$ & \\
\hline \multicolumn{12}{|c|}{ Previous psychiatric hospitalization within the last year } \\
\hline No & $41,284(58.9)$ & $39.38 \pm 45.50$ & $<.001$ & 13.2 & $<.001$ & $0.25 \pm 0.52$ & $<.001$ & $2.69 \pm 6.73$ & $<.001$ & $0.27 \pm 0.49$ & $<.001$ \\
\hline Yes & $28,852(41.1)$ & $64.35 \pm 64.90$ & & 35.8 & & $0.22 \pm 0.54$ & & $2.34 \pm 6.57$ & & $0.38 \pm 0.62$ & \\
\hline \multicolumn{12}{|c|}{ Number of psychiatric sub-diagnoses } \\
\hline $0^{a}$ & $20,867(29.7)$ & $44.41 \pm 53.51$ & $<.001$ & 23.1 & .202 & $0.17 \pm 0.45$ & $<.001$ & $1.88 \pm 5.59$ & $<.001$ & $0.14 \pm 0.41$ & $<.001$ \\
\hline $1^{\mathrm{b}}$ & $22,676(32.3)$ & $47.27 \pm 53.75$ & $d>c>b>a$ & 22.6 & & $0.22 \pm 0.50$ & $d>c>b>a$ & $2.41 \pm 6.52$ & $d>c>b>a$ & $0.31 \pm 0.54$ & $d>c>b>a$ \\
\hline $2^{c}$ & $15,471(22.1)$ & $52.35 \pm 56.76$ & & 22.9 & & $0.26 \pm 0.55$ & & $2.84 \pm 6.56$ & & $0.44 \pm 0.62$ & \\
\hline$\geq 3^{d}$ & $11,122(15.9)$ & $60.62 \pm 60.20$ & & 22.1 & & $0.36 \pm 0.64$ & & $3.67 \pm 8.54$ & & $0.48 \pm 0.66$ & \\
\hline \multicolumn{12}{|c|}{ Number of physical sub-diagnoses } \\
\hline $0^{a}$ & $22,360(31.9)$ & $42.42 \pm 53.25$ & $<.001$ & 27.0 & $<.001$ & $0.18 \pm 0.46$ & $<.001$ & $1.83 \pm 4.90$ & $<.001$ & $0.30 \pm 0.58$ & $<.001$ \\
\hline $1^{\mathrm{b}}$ & $14,091(20.1)$ & $46.03 \pm 53.94$ & $d>c>b>a$ & 23.4 & & $0.24 \pm 0.52$ & $d>c>b>a$ & $2.17 \pm 5.48$ & $d>c>b>a$ & $0.30 \pm 0.54$ & $d>a, b, c$ \\
\hline $2^{c}$ & $10,678(15.2)$ & $51.73 \pm 55.74$ & & 21.1 & & $0.26 \pm 0.55$ & & $2.66 \pm 6.96$ & & $0.29 \pm 0.51$ & \\
\hline$\geq 3^{d}$ & $23,007(32.8)$ & $57.94 \pm 57.86$ & & 18.9 & & $0.28 \pm 0.58$ & & $3.42 \pm 8.37$ & & $0.36 \pm 0.58$ & \\
\hline \multicolumn{12}{|c|}{ ECM score for the last year } \\
\hline $0^{a}$ & 7384 (10.5) & $47.88 \pm 53.18$ & $<.001$ & 13.2 & $<.001$ & $0.31 \pm 0.56$ & $<.001$ & $2.85 \pm 6.61$ & $<.001$ & $0.22 \pm 0.43$ & $<.001$ \\
\hline $1^{\mathrm{b}}$ & $9992(14.2)$ & $49.63 \pm 54.93$ & $c>a, d$ & 19.4 & & $0.23 \pm 0.51$ & $a>b, c, d$ & $2.49 \pm 6.29$ & $a>b, c, d$ & $0.23 \pm 0.46$ & $d>c>a, b$ \\
\hline $2^{c}$ & $12025(17.2)$ & $51.24 \pm 56.41$ & & 21.2 & & $0.24 \pm 0.53$ & & $2.53 \pm 6.87$ & & $0.26 \pm 0.50$ & \\
\hline$\geq 3^{d}$ & $40735(58.1)$ & $49.52 \pm 56.11$ & & 25.7 & & $0.22 \pm 0.52$ & & $2.51 \pm 6.71$ & & $0.37 \pm 0.61$ & \\
\hline \multicolumn{12}{|l|}{ Length of stay (day) } \\
\hline$<30$ & $33778(51.6)$ & - & & 21.4 & 67.72 & $0.18 \pm 0.41$ & -26.34 & $1.86 \pm 4.29$ & -27.55 & $0.32 \pm 0.55$ & 0.84 \\
\hline$\geq 30$ & $31655(48.4)$ & - & & 24.1 & $<.001$ & $0.29 \pm 0.62$ & $<.001$ & $3.25 \pm 8.40$ & $<.001$ & $0.31 \pm 0.54$ & .399 \\
\hline \multicolumn{12}{|c|}{ Insomnia sub-diagnosis } \\
\hline No & $40,934(58.4)$ & $46.29 \pm 53.89$ & $<.001$ & 21.7 & $<.001$ & $0.22 \pm 0.50$ & $<.001$ & $2.10 \pm 5.66$ & $<.001$ & $0.08 \pm 0.30$ & $<.001$ \\
\hline Yes & $29,202(41.6)$ & $54.37 \pm 57.81$ & & 24.2 & & $0.26 \pm 0.56$ & & $3.16 \pm 7.83$ & & $0.64 \pm 0.64$ & \\
\hline
\end{tabular}


Table 1. Continued

\begin{tabular}{|c|c|c|c|c|c|c|c|c|c|c|c|}
\hline \multirow[t]{2}{*}{ Variables } & \multirow[t]{2}{*}{$n(\%)$} & \multicolumn{2}{|c|}{ Length of stay } & \multicolumn{2}{|c|}{$\begin{array}{c}\text { Readmission } \\
\text { within } 30 \text { days } \\
(n=65,433)^{+}\end{array}$} & \multicolumn{2}{|c|}{$\begin{array}{c}\text { Psychiatric emergency } \\
\text { treatment }\end{array}$} & \multicolumn{2}{|c|}{$\begin{array}{l}\text { Use of injected } \\
\text { psycholeptics }\end{array}$} & \multicolumn{2}{|c|}{ Hypnotics use } \\
\hline & & $\mathrm{M} \pm \mathrm{SD}$ & $p$ & $\%$ & $p$ & $\mathrm{M} \pm \mathrm{SD}$ & $p$ & $M \pm S D$ & $p$ & $\mathrm{M} \pm \mathrm{SD}$ & $p$ \\
\hline \multicolumn{12}{|l|}{ System characteristics } \\
\hline \multicolumn{12}{|l|}{ Types of hospitals } \\
\hline Tertiarya & $2,945(4.2)$ & $23.27 \pm 19.82$ & $<.001$ & 8.7 & $<.001$ & $0.15 \pm 0.39$ & $<.001$ & $3.74 \pm 9.41$ & $<.001$ & $0.10 \pm 0.28$ & $<.001$ \\
\hline General $^{b}$ & $10,414(14.8)$ & $31.04 \pm 35.98$ & $c, d, e>b>a$ & 12.7 & & $0.16 \pm 0.45$ & $c>d>a, b . e$ & $3.00 \pm 8.10$ & $a>b>c>d, e$ & $0.20 \pm 0.42$ & $e>c>d>b>a$ \\
\hline Hospitalc & $34,553(49.3)$ & $53.85 \pm 58.34$ & & 25.4 & & $0.26 \pm 0.56$ & & $2.51 \pm 6.40$ & & $0.36 \pm 0.60$ & \\
\hline Psychiatric $^{d}$ & $18,379(26.2)$ & $55.39 \pm 59.01$ & & 24.5 & & $0.25 \pm 0.53$ & & $2.25 \pm 5.32$ & & $0.31 \pm 0.50$ & \\
\hline Clinice $^{e}$ & $3,845(5.5)$ & $55.14 \pm 61.14$ & & 27.7 & & $0.16 \pm 0.45$ & & $2.12 \pm 7.75$ & & $0.39 \pm 0.58$ & \\
\hline \multicolumn{12}{|l|}{ Number of total beds } \\
\hline $200^{\mathrm{a}}$ & $17,516(25.0)$ & $53.46 \pm 57.82$ & $<.001$ & 26.0 & $<.001$ & $0.30 \pm 0.58$ & $<.001$ & $2.59 \pm 6.90$ & $<.001$ & $0.34 \pm 0.56$ & $<.001$ \\
\hline $200 \sim 399^{b}$ & $32,801(46.8)$ & $54.20 \pm 58.59$ & $a, b>c$ & 24.6 & & $0.22 \pm 0.51$ & $a>b>c$ & $2.30 \pm 5.85$ & $c>a>b$ & $0.36 \pm 0.62$ & $b>a>c$ \\
\hline$\geq 400^{c}$ & $19,819(28.2)$ & $38.76 \pm 46.65$ & & 16.8 & & $0.20 \pm 0.50$ & & $2.91 \pm 7.65$ & & $0.22 \pm 0.44$ & \\
\hline \multicolumn{12}{|l|}{ Ownership } \\
\hline Private & $61,903(88.3)$ & $50.50 \pm 56.51$ & $<.001$ & 23.8 & $<.001$ & $0.24 \pm 0.53$ & $<.001$ & $2.54 \pm 6.67$ & .446 & $0.34 \pm 0.56$ & $<.001$ \\
\hline Public & $8,233(11.7)$ & $43.28 \pm 48.71$ & & 14.0 & & $0.18 \pm 0.51$ & & $2.60 \pm 6.69$ & & $0.15 \pm 0.37$ & \\
\hline \multicolumn{12}{|l|}{ Teaching } \\
\hline No & $57,653(82.2)$ & $52.20 \pm 57.72$ & $<.001$ & 24.9 & $<.001$ & $0.25 \pm 0.54$ & $<.001$ & $2.39 \pm 6.29$ & $<.001$ & $0.35 \pm 0.57$ & $<.001$ \\
\hline Yes & $12,483(17.8)$ & $37.90 \pm 43.27$ & & 12.7 & & $0.19 \pm 0.48$ & & $3.25 \pm 8.17$ & & $0.15 \pm 0.35$ & \\
\hline \multicolumn{12}{|l|}{ Location } \\
\hline Seoula & $8,983(12.8)$ & $45.93 \pm 50.30$ & $<.001$ & 18.5 & $<.001$ & $0.26 \pm 0.55$ & $<.001$ & $2.23 \pm 6.36$ & $<.001$ & $0.15 \pm 0.36$ & $<.001$ \\
\hline Other metropolitan ${ }^{b}$ & $20,345(29.0)$ & $46.87 \pm 54.57$ & $c>a, b$ & 24.3 & & $0.20 \pm 0.49$ & $a, c>b$ & $2.93 \pm 7.57$ & $b>a, c$ & $0.47 \pm 0.67$ & $b>c>a$ \\
\hline Province & $40,808(58.2)$ & $51.86 \pm 57.26$ & & 22.9 & & $0.25 \pm 0.54$ & & $2.42 \pm 6.23$ & & $0.27 \pm 0.49$ & \\
\hline \multicolumn{12}{|c|}{ Bed occupancy rate $(\%)$} \\
\hline$\left\langle 90.0^{\mathrm{a}}\right.$ & $30,842(44.0)$ & $53.69 \pm 57.62$ & $<.001$ & 24.1 & $<.001$ & $0.24 \pm 0.55$ & $<.001$ & $2.41 \pm 6.21$ & $<.001$ & $0.33 \pm 0.57$ & .001 \\
\hline $90.0 \sim 99.9^{b}$ & $23,463(33.4)$ & $54.72 \pm 59.98$ & $a, b>c$ & 24.4 & & $0.25 \pm 0.52$ & $a, b>c$ & $2.39 \pm 5.95$ & $c>a, b$ & $0.34 \pm 0.56$ & $a, b>c$ \\
\hline$\geq 100.0^{c}$ & $15,831(22.6)$ & $34.28 \pm 40.45$ & & 17.6 & & $0.20 \pm 0.50$ & & $3.01 \pm 8.33$ & & $0.26 \pm 0.54$ & \\
\hline \multicolumn{12}{|l|}{ RN proportion (\%) } \\
\hline$<60.0^{\mathrm{a}}$ & $32,440(46.3)$ & $55.36 \pm 59.81$ & $<.001$ & 25.3 & $<.001$ & $0.23 \pm 0.54$ & $<.001$ & $2.41 \pm 6.09$ & $<.001$ & $0.35 \pm 0.58$ & .001 \\
\hline $60.0 \sim 79.9^{b}$ & $22,022(31.4)$ & $51.75 \pm 57.01$ & $a>b>c$ & 24.6 & & $0.26 \pm 0.54$ & $b>a>c$ & $2.36 \pm 6.15$ & $c>a, b$ & $0.32 \pm 0.55$ & $a>b>c$ \\
\hline$\geq 80.0^{c}$ & $15,674(22.3)$ & $34.89 \pm 40.24$ & & 14.8 & & $0.20 \pm 0.48$ & & $3.06 \pm 8.30$ & & $0.24 \pm 0.46$ & \\
\hline \multicolumn{12}{|l|}{ System behaviors } \\
\hline \multicolumn{12}{|l|}{ Nurse staffing } \\
\hline \multicolumn{12}{|l|}{ RN-to-inpatient ratio } \\
\hline$\leq 6^{\mathrm{a}}$ & $13,051(18.6)$ & $29.60 \pm 31.34$ & $<.001$ & 10.6 & $<.001$ & $0.18 \pm 0.46$ & $<.001$ & $3.12 \pm 8.77$ & $<.001$ & $0.13 \pm 0.34$ & $<.001$ \\
\hline $6.1 \sim 13^{b}$ & $12,819(18.3)$ & $48.49 \pm 53.44$ & $c>b>a$ & 22.9 & & $0.20 \pm 0.50$ & $c>b>a$ & $2.80 \pm 7.36$ & $a>b>c$ & $0.39 \pm 0.62$ & $b>c>a$ \\
\hline$>13^{c}$ & $44,266(63.1)$ & $55.90 \pm 60.34$ & & 26.2 & & $0.26 \pm 0.55$ & & $2.30 \pm 5.65$ & & $0.35 \pm 0.58$ & \\
\hline \multicolumn{12}{|c|}{ RN-to-adj. inpatient ratio } \\
\hline$\leq 6^{\mathrm{a}}$ & $10,365(14.8)$ & $30.06 \pm 32.18$ & $<.001$ & 10.6 & $<.001$ & $0.19 \pm 0.47$ & $<.001$ & $3.14 \pm 9.11$ & $<.001$ & $0.12 \pm 0.31$ & $<.001$ \\
\hline $6.1 \sim 13^{b}$ & $10,262(14.6)$ & $44.75 \pm 49.86$ & $c>b>a$ & 19.3 & & $0.24 \pm 0.55$ & $b, c>a$ & $2.81 \pm 7.43$ & $a>b>c$ & $0.33 \pm 0.55$ & $c>b>a$ \\
\hline$>13^{c}$ & $49,509(70.6)$ & $54.77 \pm 59.59$ & & 26.0 & & $0.24 \pm 0.54$ & & $2.36 \pm 5.84$ & & $0.35 \pm 0.59$ & \\
\hline \multicolumn{12}{|c|}{ Nursing staff-to-adj. inpatient ratio } \\
\hline$\leq 6^{\mathrm{a}}$ & $11,709(16.7)$ & $33.50 \pm 37.27$ & $<.001$ & 11.3 & $<.001$ & $0.19 \pm 0.48$ & $<.001$ & $3.11 \pm 8.97$ & $<.001$ & $0.12 \pm 0.31$ & $<.001$ \\
\hline $6.1 \sim 13^{b}$ & $39,425(56.2)$ & $52.80 \pm 58.08$ & $c>b>a$ & 24.8 & & $0.25 \pm 0.54$ & $b>a, c$ & $2.43 \pm 6.01$ & $a>b, c$ & $0.36 \pm 0.59$ & $b, c>a$ \\
\hline$>13^{c}$ & $19,002(27.1)$ & $55.39 \pm 60.10$ & & 29.9 & & $0.19 \pm 0.46$ & & $2.35 \pm 7.27$ & & $0.34 \pm 0.56$ & \\
\hline
\end{tabular}

Adj.=Adjusted; $E C M=E l i x h a u s e r$ comorbidity measure; F10.x=Mental and behavioral disorders due to use of alcohol; F2.x=Schizophrenia, schizotypal and delusional disorders; F3.x=Mood disorders; $M=$ Mean; Nursing staff=total registered nurses and nurse assistants; RN=Registered nurse; $\mathrm{SD}=$ Standard deviation.

'Excluded patients who were discharged between December 2 to 31, 2016, due to unavailability of fully traceable readmissions within 30 days after discharge. 
closely related to all of the nursing staff level variables.

\section{Data analyses}

We used SAS Enterprise Guide 6.1 (SAS ver. 9.4) for all data analyses. We used descriptive statistics including frequencies, proportions, means and standard deviations, and medians and interquartile ranges (IQR) to describe patient and system characteristics. We identified the distribution of nurse staffing levels as system behaviors and patient outcomes. We used $\chi^{2}$ tests, independent t-tests, and one-way ANOVAs to examine differences in patient outcomes according to patient and system characteristics and system behaviors. Multilevel linear regression analyses were conducted to test for the associations between nurse staffing level and LOS, use of injected psycholeptics for chemical restraint, and hypnotics use. Given that LOS, injected psycholeptics, and hypnotics use all had positively skewed distributions, these variables were log-transformed to obtain an approximately normal distribution. Injected psycholeptics and hypnotics use were transformed by taking the natural log after adding 0.5 to all the values; this is because the natural log is not defined for zero values. We performed a multilevel logistic regression analysis to test for the relationship between nurse staffing level and readmission, and a multilevel ordinal logistic regression analysis was performed to examine the association between nurse staffing level and psychiatric emergency treatment. All calculated $p$-values were twosided and considered significant at $p<.05$.

\section{RESULTS}

\section{Distribution of patient and system characteristics}

The data were derived from claims of 70,136 patients across 453 institutions. Table 1 showed patient and system characteristics, and nurse staffing as system behaviors. There were more male (62.9\%) than female (37.1\%) patients, $60.9 \%$ had health insurance, and $39.1 \%$ had medical aid. The most frequent diagnosis was F10.x (37.8\%), followed by F2.x (34.6\%), and F3.x (27.6\%). Table 1 also showed univariate analyses of patient outcomes associated with each patient and system characteristic and system behavior. Outcome variables significantly differed according to all patient and system characteristics and system behaviors. Table 2 presented the system characteristics and system behaviors at the organizational level. Of the 453 institutions, most were hospitals (42.6\%), followed by psychiatric hospitals (23.2\%) and clinics (15.9\%). The median number of inpatients per RN was 16.77 $(\mathrm{IQR}=8.03)$, and the median number of adjusted inpatients per $\mathrm{RN}$ was 18.01 ( $\mathrm{IQR}=7.44)$. The median number of adjusted inpatients per nursing staff was $10.51(\mathrm{IQR}=3.09)$.

\section{Distribution of nurse staffing levels according to system characteristics}

Table 3 showed the distribution of nurse staffing levels as system behaviors according to system characteristics. Tertiary hospitals had the fewest inpatients per RN, adjusted inpatients per $\mathrm{RN}$, and adjusted inpatients per nursing staff. The number of inpatients per $\mathrm{RN}$ and adjusted inpatients per RN differed significantly according to hospital type, ownership, teaching, and location. The number of adjusted inpatients per nursing staff differed significantly according to hospital type, ownership and teaching.

\section{Distribution of health outcomes of psychiatric in- patients}

The average LOS was $49.65 \pm 55.70$ days, and readmission within 30 days was $22.8 \%$. A total of $20.2 \%$ inpatients experienced one or more psychiatric emergency treatment, with an average number of $1.17 \pm 0.55$. Nearly half $(50.2 \%)$ received at least one psycholeptic injection, with a cumulative DDD of 5.06 \pm 8.71 . A substantial proportion (40.0\%) was prescribed at least one hypnotic agent, with an average DDD of $0.74 \pm 0.62$ (Supplement 2).

\section{Relationships between nurse staffing levels and health outcomes}

We conducted multilevel analyses to examine the relationships between nurse staffing levels and health outcomes after adjusting for patient and system characteristics. All the intraclass correlation coefficients (ICCs) in the total sample were higher than 0.05 , and variances between groups or tests for $\mathrm{H}_{0}$ (random effect intercept variance $=0)$ were significant $(p=.001)$, indicating that multilevel analyses were appropriate for the data. We tested three models which included each nurse staffing variable separately: $\mathrm{RN}$-to-inpatient ratio (model $\mathrm{A}$ ), $\mathrm{RN}$-to-adjusted inpatient ratio (model B), and nursing staff-to-adjusted inpatient ratio 
Table 2. System Characteristics and System Behaviors at the Organizational Level

\begin{tabular}{|c|c|c|c|c|}
\hline Variables & Categories & $\mathrm{n}(\%)$ & $\mathrm{M} \pm \mathrm{SD}$ or Median (IQR) & Range \\
\hline \multicolumn{5}{|l|}{ System characteristics } \\
\hline \multirow{5}{*}{ Types of hospitals } & Tertiary & $17(3.7)$ & & \\
\hline & General & $66(14.6)$ & & \\
\hline & Hospital & $193(42.6)$ & & \\
\hline & Psychiatric & $105(23.2)$ & & \\
\hline & Clinic & $72(15.9)$ & & \\
\hline \multirow[t]{3}{*}{ Number of total beds } & $<200$ & $179(39.5)$ & $297.91 \pm 238.85$ & $22 \sim 1604$ \\
\hline & 200 399 & $174(38.4)$ & & \\
\hline & $\geq 400$ & $100(22.1)$ & & \\
\hline \multirow[t]{2}{*}{ Ownership } & Private & $411(90.7)$ & & \\
\hline & Public & $42(9.3)$ & & \\
\hline \multirow[t]{2}{*}{ Teaching } & No & $386(85.2)$ & & \\
\hline & Yes & $67(14.8)$ & & \\
\hline \multirow[t]{3}{*}{ Location } & Seoul & $46(10.1)$ & & \\
\hline & Other metropolitan & $124(27.4)$ & & \\
\hline & Province & $283(62.5)$ & & \\
\hline Bed occupancy rate (\%) & & & $88.3 \pm 20.4$ & 13.9 207.2 \\
\hline \multirow[t]{3}{*}{ RN proportion (\%) } & $<60.0$ & $247(54.5)$ & $58.5(23.4)$ & $16.7 \sim 100.0$ \\
\hline & $60.0 \sim 79.9$ & $121(26.7)$ & & \\
\hline & $\geq 80.0$ & $85(18.8)$ & & \\
\hline \multicolumn{5}{|l|}{ System behaviors } \\
\hline \multicolumn{5}{|l|}{ Nurse staffing } \\
\hline \multirow[t]{3}{*}{ RN-to-inpatient ratio } & $\leq 6$ & $71(15.7)$ & $16.77(8.03)$ & $0.61 \sim 45.87$ \\
\hline & $6.1 \sim 13$ & $64(14.1)$ & & \\
\hline & $>13$ & $318(70.2)$ & & \\
\hline \multirow[t]{3}{*}{ RN-to-adj. inpatient ratio } & $\leq 6$ & $56(12.4)$ & $18.01(7.44)$ & $1.24 \sim 67.07$ \\
\hline & $6.1 \sim 13$ & $52(11.5)$ & & \\
\hline & $>13$ & $345(76.1)$ & & \\
\hline \multirow[t]{3}{*}{ Nursing staff-to-adj. inpatient ratio } & $\leq 6$ & $66(14.6)$ & $10.51(3.09)$ & $1.24 \sim 33.53$ \\
\hline & $6.1 \sim 13$ & $348(76.8)$ & & \\
\hline & $>13$ & $39(8.6)$ & & \\
\hline
\end{tabular}

Adj.=Adjusted; IQR=Interquartile range; $M=$ Mean; Nursing staff=Total registered nurses and nurse assistants; RN=Registered nurse; SD=Standard deviation.

(model C). RN proportion, one of the system characteristics, was only adjusted in the multilevel Model $\mathrm{C}$. This is because the relationship between the RN proportion and the nursing staff-to-adjusted inpatient ratio was tolerable, while the $\mathrm{RN}$ proportion was closely correlated with the RN-to-inpatient ratio and the RN-toadjusted inpatient ratio. Table 4 showed the results of the multilevel analyses of Model C, where adjusted inpatients per nursing staff was used for assessing the nurse staffing level. Table 4 also included the multilevel results of other nurse staffing variables from Model B and Model C. The overall results of Model A and Model B are presented in Supplement 3 and 4, respectively. All data were included with the exception of analyses using readmis- sion data which were not complete for the entire sample. More inpatients per RN, adjusted inpatients per RN, and adjusted inpatients per nursing staff were associated with longer LOS $(B=0.02$, $p<.001 ; \mathrm{B}=0.01, p=.011 ; \mathrm{B}=0.02, p=.003$, respectively). Data from 65,433 patients were used for the readmission analysis. Having more inpatients per RN, adjusted inpatients per RN, and adjusted inpatients per nursing staff was associated with a higher risk of readmission within 30 days (OR 1.01, 95\% CI 1.00 1.02; OR 1.01, 95\% CI 1.00 1.02; OR 1.02, 95\% CI 1.00 1.04, respectively). Having more adjusted inpatients per RN and more adjusted inpatients per nursing staff was associated with lower risk of psychiatric emergency treatment (OR 0.96, 95\% CI 0.92 1.00; 
Table 3. Distribution of Nurse Staffing Levels (System Behaviors) according to System Characteristics

$(N=453)$

\begin{tabular}{|c|c|c|c|c|c|c|}
\hline \multirow{2}{*}{ Variables } & \multicolumn{2}{|c|}{ RN-to-inpatient ratio } & \multicolumn{2}{|c|}{ RN-to-adj. inpatient ratio } & \multicolumn{2}{|c|}{ Nursing staff-to-adj. inpatient ratio } \\
\hline & Median (IQR) & Range & Median (IQR) & Range & Median (IQR) & Range \\
\hline \multicolumn{7}{|l|}{ Types of hospitals } \\
\hline Tertiarya & $1.48(0.91)$ & $0.78 \sim 4.10$ & $3.97(1.74)$ & $1.95 \sim 11.11$ & $3.56(1.76)$ & $1.92 \sim 9.54$ \\
\hline General $^{\mathrm{b}}$ & $3.37(7.95)$ & $0.61 \sim 24.63$ & $6.14(6.23)$ & $2.11 \sim 26.97$ & $5.87(4.52)$ & $2.08 \sim 16.18$ \\
\hline Hospitak & $17.88(5.65)$ & $0.67 \sim 29.41$ & $18.51(5.61)$ & $1.24 \sim 31.06$ & $10.86(1.88)$ & $1.24 \sim 15.53$ \\
\hline Psychiatric $^{d}$ & $17.31(4.04)$ & $2.28 \sim 23.68$ & $18.36(4.14)$ & $4.20 \sim 24.24$ & $10.51(2.12)$ & $3.80 \sim 13.32$ \\
\hline Clinice $^{e}$ & $19.75(8.80)$ & $1.36 \sim 45.87$ & $22.50(7.36)$ & $6.23 \sim 67.07$ & $11.81(3.48)$ & $4.32 \sim 33.53$ \\
\hline$p$ & \multicolumn{2}{|c|}{$\begin{array}{c}\langle .001 \\
c, d, e>a, b\end{array}$} & \multicolumn{2}{|c|}{$\begin{array}{c}\langle .001 \\
e>c, d>a, b\end{array}$} & \multicolumn{2}{|c|}{$\begin{array}{c}<.001 \\
c, d, e>a, b\end{array}$} \\
\hline \multicolumn{7}{|l|}{ Ownership } \\
\hline Private & $17.15(6.92)$ & $0.78 \sim 45.87$ & $18.45(6.49)$ & $1.95 \sim 67.07$ & $10.67(2.79)$ & $1.92 \sim 33.53$ \\
\hline Public & $4.31(10.47)$ & $0.61 \sim 19.65$ & $6.28(9.33)$ & $1.24 \sim 19.88$ & $5.57(5.69)$ & $1.24 \sim 11.81$ \\
\hline$p$ & \multicolumn{2}{|c|}{$<.001$} & \multicolumn{2}{|c|}{$<.001$} & \multicolumn{2}{|c|}{$<.001$} \\
\hline \multicolumn{7}{|l|}{ Teaching } \\
\hline No & $17.49(6.35)$ & $0.61 \sim 45.87$ & $18.75(6.07)$ & $2.11 \sim 67.07$ & $10.80(2.50)$ & $2.08 \sim 33.53$ \\
\hline Yes & $2.57(5.04)$ & $0.67 \sim 19.65$ & $5.53(6.18)$ & $1.24 \sim 19.88$ & $4.80(4.49)$ & $1.24 \sim 11.81$ \\
\hline$p$ & \multicolumn{2}{|c|}{$<.001$} & \multicolumn{2}{|c|}{$<.001$} & \multicolumn{2}{|c|}{$<.001$} \\
\hline \multicolumn{7}{|l|}{ Location } \\
\hline Seoula & $11.96(14.83)$ & $0.67 \sim 41.92$ & $12.19(15.78)$ & $1.24 \sim 67.07$ & $10.07(7.47)$ & $1.24 \sim 33.53$ \\
\hline Other metropolitan ${ }^{b}$ & $16.02(7.56)$ & $0.99 \sim 29.41$ & $17.77(7.25)$ & $2.14 \sim 31.06$ & $10.23(3.16)$ & $1.92 \sim 15.53$ \\
\hline Province ${ }^{c}$ & $17.28(7.68)$ & $0.61 \sim 45.87$ & $18.45(6.38)$ & $1.95 \sim 53.21$ & $10.74(2.88)$ & $1.95 \sim 26.60$ \\
\hline$p$ & \multicolumn{2}{|c|}{$<.001$} & \multicolumn{2}{|c|}{.017} & \multicolumn{2}{|c|}{.185} \\
\hline
\end{tabular}

Adj.=Adjusted; IQR=Interquartile range; Nursing staff=Total registered nurses and nurse assistants; RN=Registered nurse.

OR 0.92, 95\% CI 0.84 1.00, respectively). These same conditions (more adjusted inpatients per RN and more adjusted inpatients per nursing staff) were also associated with greater hypnotics use $[B=0.00$ (rounded off to the second digit after the decimal point, and bigger than 0.00), $p=.048 ; \mathrm{B}=0.01, p=.028$, respectively]. Nurse staffing level was not significantly associated with use of injected psycholeptics.

\section{DISCUSSION}

The median number of inpatients per RN, adjusted inpatients per RN, and adjusted inpatients per nursing staff in psychiatric wards were 16.77, 18.01, and 10.51, respectively. In terms of hospital type, only psychiatric wards in tertiary and general hospitals met the Korean legal standards for the $\mathrm{RN}$-to-inpatient ratio (1.48 and 3.37, respectively) and $\mathrm{RN}-$ to-adjusted inpatient ratio (3.97 and 6.14, respectively), while nursing staff-to-adjusted inpatient ratio for all types of hospitals met appropriate expectations. However, when comparing the current nursing staff-to-adjusted inpatient ratio with Japanese legal standards for the number of psychiatric beds [8,9], only tertiary hospitals showed a similar staffing level (3.56). The U.S. nurse staffing standard (i.e., California legal standard) is expressed as the licensed nurse-to-patient ratio during one shift [7]. Kim et al. [17] suggested that the U.S. standard of 1:6 for acute psychiatric wards can be converted to an employed nurse-to-patient ratio (a ratio which includes administrative nurses, nurses working with outpatients, and offduty nurses) of 1:1.25 in Korea. Thus, when compared to the U.S., all types of hospitals had insufficient nursing staff in psychiatric wards. Additionally, the number of nurses included in the present study counted ward managers and nurses working in psychiatric outpatient and daycare wards, not just psychiatric inpatient wards. Thus, in practice, the number of inpatients per $\mathrm{RN}$ during one shift may actually be higher than what was reported in the present study.

According to the Korean legal standard for psychiatric wards, the number of employed nurses (not equal to the number of nurses during one shift) is used to calculate the nurse-to-patient 
Table 4. Results of Multilevel Analyses Predicting Health Outcomes

\begin{tabular}{|c|c|c|c|c|c|c|c|c|c|c|c|c|c|c|}
\hline \multirow[t]{2}{*}{ Variables } & \multirow[t]{2}{*}{ Model } & \multicolumn{3}{|c|}{$\begin{array}{l}\text { Length of stay } y^{+\varsigma} \\
\qquad(n=70,136)\end{array}$} & \multicolumn{2}{|c|}{$\begin{array}{l}\text { Readmission within } \\
30 \text { days }(n=65,433)^{++}\end{array}$} & \multicolumn{2}{|c|}{$\begin{array}{l}\text { Psychiatric emergency } \\
\text { treatment }(n=70,136)\end{array}$} & \multicolumn{3}{|c|}{$\begin{array}{l}\text { Use of injected } \\
\text { psycholeptics } \\
(n=70,136)\end{array}$} & \multicolumn{3}{|c|}{$\begin{array}{l}\text { Hypnotics use } \\
\qquad(n=70,136)\end{array}$} \\
\hline & & Estimate & SE & $p$ & OR & $95 \% \mathrm{Cl}$ & OR & $95 \% \mathrm{Cl}$ & Estimate & SE & $p$ & Estimate & SE & $p$ \\
\hline \multicolumn{15}{|l|}{ Patient characteristics } \\
\hline Age & $C$ & 0.00 & 0.00 & $<.001$ & $0.99^{* * *}$ & $0.99 \sim 0.99$ & $0.99^{* * *}$ & $0.98 \sim 0.99$ & -0.01 & 0.00 & $<.001$ & $0.00^{\prime \prime}$ & 0.00 & $<.001$ \\
\hline Gender (ref. male) & $C$ & -0.04 & 0.01 & $<.001$ & $0.89^{* * *}$ & $0.85 \sim 0.93$ & $0.94^{*}$ & $0.89 \sim 0.99$ & 0.05 & 0.01 & $<.001$ & -0.01 & 0.00 & .027 \\
\hline Insurance (ref. medical aid) & C & -0.41 & 0.01 & $<.001$ & $0.65^{* * *}$ & $0.61 \sim 0.68$ & $1.60^{\star * *}$ & $1.52 \sim 1.68$ & 0.11 & 0.01 & $<.001$ & -0.07 & 0.00 & $<.001$ \\
\hline \multicolumn{15}{|l|}{ Diagnosis (ref. F10.x) } \\
\hline$F 2 . x$ & $C$ & 0.49 & 0.01 & $<.001$ & $0.92^{* *}$ & $0.87 \sim 0.97$ & $0.73^{* * *}$ & $0.68 \sim 0.77$ & -0.25 & 0.01 & $<.001$ & -0.07 & 0.00 & $<.001$ \\
\hline$F 3 . x$ & $C$ & 0.10 & 0.01 & $<.001$ & $0.79^{* * *}$ & $0.75 \sim 0.84$ & $0.69^{* * *}$ & $0.64 \sim 0.73$ & -0.23 & 0.01 & $<.001$ & -0.02 & 0.00 & $<.001$ \\
\hline $\begin{array}{l}\text { Previous psychiatric } \\
\text { hospitalization within the } \\
\text { last year (ref. no) }\end{array}$ & C & 0.41 & 0.01 & $<.001$ & $2.74^{* * *}$ & $2.62 \sim 2.86$ & $0.75^{* * *}$ & $0.71 \sim 0.79$ & -0.13 & 0.01 & $<.001$ & 0.02 & 0.00 & $<.001$ \\
\hline $\begin{array}{l}\text { Number of psychiatric } \\
\text { sub-diagnoses }\end{array}$ & C & 0.17 & 0.00 & $<.001$ & 1.01 & $0.99 \sim 1.03$ & $1.13^{* * *}$ & $1.10 \sim 1.15$ & 0.11 & 0.00 & $<.001$ & 0.02 & 0.00 & $<.001$ \\
\hline $\begin{array}{l}\text { Number of physical } \\
\text { sub-diagnoses }\end{array}$ & C & 0.15 & 0.00 & $<.001$ & 0.99 & $0.98 \sim 1.00$ & $1.06^{* * *}$ & $1.05 \sim 1.08$ & 0.04 & 0.00 & $<.001$ & $0.00^{\| \prime}$ & 0.00 & $<.001$ \\
\hline ECM score for the last year & C & -0.05 & 0.00 & $<.001$ & $1.03^{* * *}$ & $1.02 \sim 1.04$ & $0.97^{\star \star *}$ & $0.96 \sim 0.98$ & $0.00^{\prime \prime}$ & 0.00 & .144 & 0.01 & 0.00 & $<.001$ \\
\hline Length of stay $\geq 30$ (ref. <30) & C & - & - & - & $0.92^{* * *}$ & $0.88 \sim 0.96$ & $1.47^{\star * *}$ & $1.40 \sim 1.54$ & 0.22 & 0.01 & $<.001$ & -0.03 & 0.00 & $<.001$ \\
\hline $\begin{array}{l}\text { Insomnia sub-diagnosis } \\
\text { (ref. no) }\end{array}$ & C & - & - & - & - & - & - & - & - & - & - & 0.52 & 0.00 & $<.001$ \\
\hline \multicolumn{15}{|l|}{ System characteristics } \\
\hline \multicolumn{15}{|l|}{ Types of hospitals (ref. tertiary) } \\
\hline General & $\mathrm{C}$ & -0.01 & 0.11 & .910 & 1.12 & $0.86 \sim 1.45$ & 0.99 & $0.29 \sim 3.36$ & -0.14 & 0.10 & .169 & 0.04 & 0.05 & .468 \\
\hline Hospital & C & 0.28 & 0.13 & .031 & $1.55^{\star *}$ & $1.14 \sim 2.11$ & 1.64 & $0.37 \sim 7.32$ & -0.22 & 0.13 & .081 & 0.02 & 0.07 & .767 \\
\hline Psychiatric & c & 0.39 & 0.13 & .002 & $1.58^{* *}$ & $1.16 \sim 2.15$ & 1.63 & $0.36 \sim 7.36$ & -0.22 & 0.13 & .082 & 0.01 & 0.07 & .892 \\
\hline Clinic & c & 0.25 & 0.15 & .106 & $1.70^{\star *}$ & $1.17 \sim 2.46$ & 1.02 & $0.17 \sim 6.34$ & -0.44 & 0.15 & .003 & -0.01 & 0.08 & .935 \\
\hline Number of total beds & c & 0.00 & 0.00 & .717 & 1.00 & $1.00 \sim 1.00$ & 1.00 & $1.00 \sim 1.00$ & 0.00 & 0.00 & .782 & $0.00^{\| \prime}$ & 0.00 & .944 \\
\hline Ownership (ref. private) & C & 0.11 & 0.07 & .097 & 0.98 & $0.83 \sim 1.16$ & 0.76 & $0.34 \sim 1.71$ & -0.20 & 0.07 & .003 & 0.01 & 0.04 & .756 \\
\hline Teaching (ref. no) & C & 0.03 & 0.07 & .631 & $0.80^{* *}$ & $0.68 \sim 0.94$ & 0.87 & $0.40 \sim 1.92$ & 0.10 & 0.07 & .142 & -0.04 & 0.03 & .197 \\
\hline \multicolumn{15}{|l|}{ Location (ref. Seoul) } \\
\hline Other metropolitan & C & -0.09 & 0.07 & .200 & 1.10 & $0.94 \sim 1.28$ & 0.69 & $0.31 \sim 1.49$ & 0.18 & 0.07 & .005 & 0.13 & 0.03 & $<.001$ \\
\hline Province & C & -0.04 & 0.06 & .575 & 1.04 & $0.90 \sim 1.20$ & 0.62 & $0.30 \sim 1.28$ & 0.04 & 0.06 & .512 & 0.01 & 0.03 & .732 \\
\hline Bed occupancy rate & c & $0.00^{\prime \prime}$ & 0.00 & .059 & 1.00 & $1.00 \sim 1.00$ & 1.00 & $0.99 \sim 1.01$ & 0.00 & 0.00 & .777 & $0.00^{\prime \prime}$ & 0.00 & .238 \\
\hline RN proportion & C & $0.00^{\|}$ & 0.00 & .982 & 1.00 & $1.00 \sim 1.00$ & 1.01 & $0.98 \sim 1.01$ & $0.00^{\prime \prime}$ & 0.00 & .052 & 0.00 & 0.00 & .971 \\
\hline \multicolumn{15}{|l|}{ System behaviors } \\
\hline \multicolumn{15}{|l|}{ Nurse staffing } \\
\hline RN-to-inpatient ratio & A & 0.02 & 0.00 & $<.001$ & $1.01^{*}$ & $1.00 \sim 1.02$ & 0.97 & $0.93 \sim 1.02$ & 0.00 & 0.00 & .244 & 0.00 & 0.00 & .212 \\
\hline RN-to-adj. inpatient ratio & B & 0.01 & 0.00 & .011 & $1.01^{*}$ & $1.00 \sim 1.02$ & $0.96^{*}$ & $0.92 \sim 1.00$ & 0.00 & 0.00 & .413 & 0.00 & 0.00 & .048 \\
\hline $\begin{array}{l}\text { Nursing staff-to-adj. } \\
\text { inpatient ratio }\end{array}$ & C & 0.02 & 0.01 & .003 & $1.02^{* *}$ & $1.00 \sim 1.04$ & $0.92^{*}$ & $0.84 \sim 1.00$ & $0.00^{\prime \prime}$ & 0.01 & .783 & 0.01 & 0.00 & .028 \\
\hline
\end{tabular}

Adj.=Adjusted; $\mathrm{Cl}=$ Confidence interval; $\mathrm{ECM}=$ Elixhauser comorbidity measures; $\mathrm{F10.x=Mental}$ and behavioral disorders due to use of alcohol; F2.X=Schizophrenia, schizotypal and delusional disorders; F3.x=Mood disorders; Model A includes RN-to-inpatient ratio as the nurse staffing variable; Model B includes RN-to-adjusted inpatient ratio as the nurse staffing variable; Model $\mathrm{C}$ includes nursing staff-to-adjusted inpatient ratio as the nurse staffing variable; Nursing staff=total registered nurses and nurse assistants; OR=Odds ratio; ref.=Reference; RN=Registered nurse; $\mathrm{SE}=$ Standard error.

${ }^{+}$Log transformation; ${ }^{+}$Excluded patients who were discharged between December 2 to 31, 2016, due to unavailability of fully traceable readmissions within 30 days after discharge; ${ }^{\S}$ Values of 0.00 are not exactly equal to zero; "Values are lower than 0.00.

${ }^{*} p<.05 ;{ }^{* *} p<.01 ;{ }^{* *} p<.001$. 
ratio, and nurses can be replaced by nurse assistants within half of the registered nurse capacity [6]. This condition might exacerbate the qualitative deviations in the nurse staffing levels by hospital type and shift in clinical practice. For instance, it is possible to have only nursing assistants assigned to a shift within hospitals with low nurse staffing levels [17]. Therefore, the nurse-to-patient ratio per shift, which is presented in detail according to the type of hospital, should be prepared in domestic legal standard.

Based on the multilevel modeling analyses, nurse staffing variables were significantly associated with five health outcomes among inpatients. First, more inpatients per RN, adjusted inpatients per RN, and adjusted inpatients per nursing staff were associated with longer LOS. This result is similar to previous findings reporting significant LOS differences relative to nurse-topatient ratios in psychiatric units. Specifically, fewer patients per nurse was previously related to a reduced LOS [17], and having more nurses in general hospital psychiatric units was associated with shorter LOS [16]. However, it should be noted that measures used to determine nurse staffing levels in previous studies differ from what was used in the current study [16,17]. To help psychiatric inpatients return to the community, it is important to reduce hospitalization length by providing improved quality of care and promoting stabilization and recovery [17]. Nurses' ongoing observations and assessments, recognition, interpretations of clinical data, and decision-making were found an important factor for patient recovery $[24,41]$. Sufficient nurse staffing was previously shown to enable effective surveillance and interventions, contributing to recovery and reduced LOS [24,42].

The second significant finding was that more inpatients per $\mathrm{RN}$, adjusted inpatients per RN, and adjusted inpatients per nursing staff were also associated with a higher risk of readmission within 30 days. The current results are similar to a previous finding, which reported that the number of nurses had an inverse association with readmission risk within 30 days [18]. Mental health nurses need to develop patient-centered recovery strategies and care plans during the transition from the hospital back into the community, while providing nursing support for patients' recovery through medication management, patient education, discharge plans, interpersonal effectiveness, and practical daily living [23,43]. In particular, early non-adherence to medication after discharge was a crucial factor influencing readmission [44]. Hence, in order to reduce readmissions in patients with mental illnesses, hospitals should make effort to have a sufficient nurse staffing.

Third, more adjusted inpatients per RN and adjusted inpatients per nursing staff were also associated with less psychiatric emergency treatment, which corresponded to a diminished need to use seclusion/restraints. That is, a lower nurse staffing level was associated with a lower risk of psychiatric emergency treatment. Previous studies investigating the relationship between nurse staffing and seclusion or restraint use reported inconsistent results. Specifically, while De Lacy [22] reported that more nursing staff within psychiatric wards was related to fewer instances of using seclusion and restraints, Janssen et al. [19] found these variables to be unrelated. Fukasawa et al. [21], on the other hand, reported that more nurses per bed were associated with an increased use of seclusion and mechanical restraints. In contrast to common expectations regarding nurses' roles and abilities in reducing the use of restraints by employing knowledge and skills to meet patient needs and engage as active partners in the treatment process [25], the current study observed that lower nurse staffing levels were associated with less psychiatric emergency treatment. This might be due to insufficient adjustments to understanding patient severity regarding the nature of administrative data. It could also be that patients who were anticipated to need coercive measures, such as seclusion and restraints, tended to be admitted to wards that were assigned more nurses [21]. In general, hospitals with patients in less severe conditions tended to have lower nurse staffing levels [45]. Thus, hospitals with patients exhibiting a high severity of acute symptoms may employ more nurses to enable proper treatment and coping.

In the present study, we examined only the raw nurse staffing level without examining the qualitative characteristics of nurses' educational and work experience. Previous studies reported that nurse staffing, nursing work environments, and nurse education were all associated with patient outcomes $[19,46]$. Thus, the development of a measurement tool that can properly adjust for inpatient severity is necessary and further studies are needed to investigate the relationship between nurse staffing and the use of seclusion and restraints. 
The fourth outcome of the present study found that the relationship between the nurse staffing level and injected psycholeptics for chemical restraint was not significant. Although the influence of nurse staffing on the use of chemical restraints has rarely been investigated, it is expected that mental health nurses could help patients stabilize and reduce the use of chemical restraints $[22,25]$. However, we found that the nurse staffing level was not associated with use of injected psycholeptics. The current result could be interpreted in two ways. First, patient characteristics, such as diagnosis and symptom severity, may have greater effects on the use of injected psycholeptics than do nurse staffing levels. Alternatively, the nurse staffing level may affect the use of injected psycholeptics, but significant relationship was not confirmed when not accounting for the qualitative aspects of the nursing staff present. Further studies should consider proper adjustments based on patient severity and measurement of both quantitative and qualitative aspects of nurse staffing levels. Moreover, we suggest that a longitudinal study is needed to analyze the influence of the nurse staffing level on changes to the number of mechanical and chemical restraints simultaneously employed.

Fifth, more adjusted inpatients per $\mathrm{RN}$ and adjusted inpatients per nursing staff were also associated with an increased use of hypnotics. Nurses were reported to often be the first to recognize sleep-related problems in patients helping them manage sleep disturbances through behavioral modifications and stimulus control $[29,47]$. A previous study found that a sleep enhancement care as the nursing intervention for psychiatric inpatients was effective in improving subjective sleep quality, although patient consumption of psycho-active medications did not change between admission and discharge [47]. According to the Nursing Intervention Classifications, nurses may provide sleep enhancement care by assessing patterns of sleep and activities, sleep hygiene education, promoting daytime activities, and applying progressive muscle relaxation or massage [48]. Therefore, sufficient staffing may allow nurses to provide tailored sleep enhancement care to patient possibly contributing to the prevention the excessive use of hypnotics.

In the present study, the relationship between nurse staffing and patient outcomes showed similar patterns regardless of the type of nurse staffing (i.e., RN vs. nursing staff). These results might suggest that patient outcomes in this study were not closely related to nursing interventions for conditions such as urinary tract infection and pneumonia [49,50]. Therefore, future research is needed to investigate the differences in the influences of number of patients per $\mathrm{RN}$ and number of patients per nursing staff on nursing-sensitive outcomes in psychiatric wards. Further, research is needed to identify the optimal nurse staffing levels and nursing skill mix to improve psychiatric health outcomes.

This study examined the relationship between nurse staffing level and psychiatric health outcomes based on PCDM thus contributing to the body of knowledge on the importance of nurse staffing in meeting patient needs and achieving positive health outcomes. The current findings also provided evidence that can be used for reviewing domestic legal standards and establishing policies on the nurse staffing in psychiatric wards.

A few study limitations should be noted. First, hospitals that did not submit estimation reports on the differentiating policies for hospitalization fees in medical aid psychiatric departments were not included in this study. Out of the initial 710 institutions that claimed reimbursement for psychiatric inpatient services to inpatients with primary diagnoses of F10.x, F2.x, or F3.x in 2016, approximately 200 institutions did not include staffing information. Therefore, one might argue that the present study did not provide an accurate overview of the nurse staffing ratios within the Korean healthcare system. Second, the nurse staffing level reported in the current study may not be completely accurate, as the number of RNs sampled included administrative nurses (i.e., outpatient and daycare nurses and ward managers). Thus, the nurse staffing level presented in this study might not be the same as the actual level in practice. Third, adjusting for patient severity while using National Health Insurance claims data was insufficient given that we were limited in our access to the available information and variables. Fourth, some hospitalizations and readmissions due primarily to the lack of family and social support systems may have been included, because it is not possible to screen hospitalization episodes using administrative data. These cases are likely to result in longer LOS or more readmissions. Fifth, since the severity and characteristics of psychiatric inpatients and the function of hospitals may differ depending on the type of hospital, the results of this study may not be generalized to all types of psychiatric hospitals. Hence, future studies should con- 
sider hospital type and examine hospitals with similar functions and having patients with comparable characteristics and symptom severity in their psychiatric wards. Sixth, non-benefit medications were not included in the National Health Insurance claims data.

\section{CONCLUSION}

This study investigated the association between nurse staffing level and psychiatric health outcomes based on PCDM. The current study found that nurse staffing levels were associated with health outcomes of psychiatric inpatients. Particularly, a lower nurse staffing level was related to a longer LOS, higher risk of readmission within 30 days, and increased hypnotics use. To improve the health outcomes of psychiatric inpatients, adjustments need to be made to increase the nurse staffing level in psychiatric wards as prescribed in the existing national law by establishing detailed standards according to shift work and the type of hospital. A review of domestic legal standards is needed to improve psychiatric nurse staffing levels and enact policies for optimizing nurse staffing in Korea.

\section{CONFLICTS OF INTEREST}

The authors declared no conflict of interest.

\section{AUTHOR CONTRIBUTIONS}

Conceptualization or/and Methodology: Park Suin \& Kim S.

Data curation or/and Analysis: Park Suin \& Park Sohee.

Project administration or/and Supervision: Lee YJ \& Jung YC \& Kim S.

Resources or/and Software: Park CS.

Validation: Kim S \& Park Sohee \& Lee YJ \& Park CS \& Jung YC \& Park Suin.

Writing original draft or/and Review \& editing: Park Suin \& Kim S \& Park Sohee \& Lee YJ \& Park CS \& Jung YC.

\section{REFERENCES}

1. Organisation for Economic Co-operation and Development
(OECD). Hospital average length of stay by diagnostic categories [Internet]. Paris: OECD; c2020 [cited 2020 May 14]. Available from: http://stats.oecd.org/.

2. Health Insurance Review \& Assessment Service (HIRA). OECD health statistics comparison database in 2014 [Internet]. Wonju: HIRA; c2014 [cited 2020 May 14]. Available from: http://www.hira.or.kr/bbsDummy.do?pgmid $=$ HIRAA020045010000\&brdScnBltNo $=4 \&$ brdBlt No $=2274 \&$ pageIndex $=1 \#$ none.

3. Health Insurance Review \& Assessment Service (HIRA). Psychiatric emergency treatment [Internet]. Wonju: HIRA; c2020 [cited 2020 May 14]. Available from: http://opendata.hira. or.kr/op/opc/olapDiagBhvInfo.do.

4. Health Insurance Review \& Assessment Service (HIRA). Hypnotics and sedatives use [Internet]. Wonju: HIRA; c2020 [cited 2020 May 14]. Available from: http://opendata.hira.or.kr/op/ opc/olapMeftDiv.do.

5. Health Insurance Review \& Assessment Service (HIRA). Behavior definition of relative value score - psychiatric emergency treatment [Internet]. Wonju: HIRA; c2020 [cited 2020 May 14]. Available from: https://biz.hira.or.kr/index.do?sso = ok.

6. Ministry of Health and Welfare. Enforcement rule of the act on the improvement of mental health and the support for welfare services for mental patients, article 11, paragraph 2 [Internet]. Sejong: Ministry of Government Legislation; c2017 [cited 2020 May 14]. Available from: http://www.law.go.kr/lsInfoP. do? 1 siSeq $=206272 \&$ efYd $=20190101 \# 0000$.

7. California Office of Administrative Law. Nursing service staff, 22 CA ADC § 70217 [Internet]. Toronto: Thomson Reuters Westlaw; c2004 [cited 2020 May 14]. Available from: https://govt.westlaw.com/calregs/Document/I8612C410941F11E29091E6B951DDF6CE? view Type = FullText\&originationContext $=$ documenttoc $\&$ transition Type $=$ CategoryPage Item\&contextData $=($ sc. Default $)$.

8. Morioka N, Tomio J, Seto T, Kobayashi Y. The association between higher nurse staffing standards in the fee schedules and the geographic distribution of hospital nurses: A cross-sectional study using nationwide administrative data. BMC Nursing. 2017;16:25. https://doi.org/10.1186/s12912-017-0219-1

9. You SJ. Policy implications of nurse staffing legislation. The Journal of the Korea Contents Association. 2013;13(6) :380389. https://doi.org/10.5392/JKCA.2013.13.06.380

10. Pitkäaho T, Partanen $\mathrm{P}$, Miettinen $\mathrm{MH}$, Vehviläinen-Julkunen $\mathrm{K}$. The relationship between nurse staffing and length of stay in acute-care: A one-year time-series data. Journal of Nursing Management. 2016;24(5):571-579.

https://doi.org/10.1111/jonm.12359

11. Cho E, Park J, Choi M, Lee HS, Kim EY. Associations of nurse staffing and education with the length of stay of surgical patients. Journal of Nursing Scholarship. 2018;50(2):210-218. 
https://doi.org/10.1111/jnu.12366

12. Lasater KB, Mchugh MD. Nurse staffing and the work environment linked to readmissions among older adults following elective total hip and knee replacement. International Journal for Quality in Health Care. 2016;28(2):253-258.

https://doi.org/10.1093/intqhc/mzw007

13. Kim SJ, Park EC, Han KT, Kim SJ, Kim TH. Nurse staffing and 30-day readmission of chronic obstructive pulmonary disease patients: A 10-year retrospective study of patient hospitalization. Asian Nursing Research. 2016;10(4):283-288. https://doi.org/10.1016/j.anr.2016.09.003

14. Driscoll A, Grant MJ, Carroll D, Dalton S, Deaton C, Jones I, et al. The effect of nurse-to-patient ratios on nurse-sensitive patient outcomes in acute specialist units: A systematic review and meta-analysis. European Journal of Cardiovascular Nursing. 2018;17(1):6-22. https://doi.org/10.1177/1474515117721561

15. Cho E, Chin DL, Kim S, Hong O. The relationships of nurse staffing level and work environment with patient adverse events. Journal of Nursing Scholarship. 2016;48(1):74-82. https://doi.org/10.1111/jnu.12183

16. Pertile R, Donisi V, Grigoletti L, Angelozzi A, Zamengo G, Zulian G, et al. DRGs and other patient-, service- and area-level factors influencing length of stay in acute psychiatric wards: The Veneto region experience. Social Psychiatry and Psychiatric Epidemiology. 2011;46(7):651-660.

https://doi.org/10.1007/s00127-010-0231-1

17. Kim GH, Kim SJ, Kim SS. Nurse staffing, inpatient's length of stay and safety accidents in psychiatric hospital settings. Journal of the Korean Data Analysis Society. 2017;19(1):503-511.

18. Han KT, Kim SJ, Jang SI, Hahm MI, Kim SJ, Lee SY, et al. The outcomes of psychiatric inpatients by proportion of experienced psychiatrists and nurse staffing in hospital: New findings on improving the quality of mental health care in South Korea. Psychiatry Research. 2015;229(3):880-886.

https://doi.org/10.1016/j.psychres.2015.07.051

19. Janssen W, Noorthoorn E, van Linge R, Lendemeijer B. The influence of staffing levels on the use of seclusion. International Journal of Law and Psychiatry. 2007;30(2):118-126. https://doi.org/10.1016/j.ijlp.2006.04.005

20. Donat DC. Impact of improved staffing on seclusion/restraint reliance in a public psychiatric hospital. Psychiatric Rehabilitation Journal. 2002;25(4):413-416. https://doi.org/10.1037/h0094994

21. Fukasawa M, Miyake M, Suzuki Y, Fukuda Y, Yamanouchi Y. Relationship between the use of seclusion and mechanical restraint and the nurse-bed ratio in psychiatric wards in Japan. International Journal of Law and Psychiatry. 2018;60:57-63. https://doi.org/10.1016/j.ijlp.2018.08.001

22. De Lacy LC. The influence of nursing staff numbers and skill mix on seclusion and restraint use in public psychiatric hospi- tals [dissertation]. Fairfax (VA): George Mason University; 2006. p. 1-157.

23. McHugh MD, Ma C. Hospital nursing and 30-day readmissions among medicare patients with heart failure, acute myocardial infarction, and pneumonia. Medical Care. 2013;51(1):52-59. https://doi.org/10.1097/MLR.0b013e3182763284

24. Kutney-Lee A, Lake ET, Aiken LH. Development of the hospital nurse surveillance capacity profile. Research in Nursing \& Health. 2009;32(2):217-228. https://doi.org/10.1002/nur.20316

25. Cusack P, Cusack FP, McAndrew S, McKeown M, Duxbury J. An integrative review exploring the physical and psychological harm inherent in using restraint in mental health inpatient settings. International Journal of Mental Health Nursing. 2018;27(3):1162-1176. https://doi.org/10.1111/inm.12432

26. Masters KJ, Bellonci C, Bernet W, Arnold V, Beitchman J, Benson RS, et al. Practice parameter for the prevention and management of aggressive behavior in child and adolescent psychiatric institutions, with special reference to seclusion and restraint. Journal of the American Academy of Child and Adolescent Psychiatry. 2002;41(2 Suppl) :4S-25S. https://doi.org/10.1097/00004583-200202001-00002

27. Müller MJ, Olschinski C, Kundermann B, Cabanel N. Subjective sleep quality and sleep duration of patients in a psychiatric hospital. Sleep Science. 2016;9(3):202-206.

https://doi.org/10.1016/j.slsci.2016.08.004

28. Lee MJ, Hah YS. The effect of relaxation training applied to psychiatric inpatients complaining of insomnia. Journal of Korean Academy of Psychiatric Mental Health Nursing. 2008;17(1):35-45.

29. Pellatt GC. The nurse's role in promoting a good night's sleep for patients. British Journal of Nursing. 2007;16(10):602-605. https://doi.org/10.12968/bjon.2007.16.10.23507

30. O'Brien-Pallas L, Meyer RM, Hayes LJ, Wang S. The patient care delivery model--an open system framework: Conceptualisation, literature review and analytical strategy. Journal of Clinical Nursing. 2011;20(11-12):1640-1650. https://doi.org/10.1111/j.1365-2702.2010.03391.x

31. Reitan SK, Helvik AS, Iversen V. Use of mechanical and pharmacological restraint over an eight-year period and its relation to clinical factors. Nordic Journal of Psychiatry. 2018;72(1):24-30. https://doi.org/10.1080/08039488.2017.1373854

32. WHO Collaborating Centre for Drug Statistics Methodology. Guidelines for ATC classification and DDD assignment 2018. Oslo: WHO Collaborating Centre for Drug Statistics Methodology; 2017. p. 22-30, 223-226.

33. Ministry of Food and Drug Safety. Regulation on codes for classification of drugs and other products [Internet]. Cheongju: Sejong: Ministry of Government Legislation; c2009 [cited 
2020 May 14]. Available from: http://www.law.go.kr/admRulLsInfoP.do? admRulSeq $=2100000038481$.

34. Okumura Y, Sugiyama N, Noda T, Sakata N. Association of high psychiatrist staffing with prolonged hospitalization, follow-up visits, and readmission in acute psychiatric units: A retrospective cohort study using a nationwide claims database. Neuropsychiatric Disease and Treatment. 2018;14:893-902. https://doi.org/10.2147/NDT.S160176

35. Šprah L, Dernovšek MZ, Wahlbeck K, Haaramo P. Psychiatric readmissions and their association with physical comorbidity: A systematic literature review. BMC Psychiatry. 2017;17(1):2. https://doi.org/10.1186/s12888-016-1172-3

36. Donisi V, Tedeschi F, Wahlbeck K, Haaramo P, Amaddeo F. Pre-discharge factors predicting readmissions of psychiatric patients: A systematic review of the literature. BMC Psychiatry. 2016;16(1):449. https://doi.org/10.1186/s12888-016-1114-0

37. Cha SK, Kim SS. The determinant of the length of stay in hospital for schizophrenic patients: Using data from the in-depth injury patient surveillance system. Journal of Digital Convergence. 2013;11(4):351-359. https://doi.org/10.14400/JDPM.2013.11.4.351

38. Knutzen M, Bjørkly S, Eidhammer G, Lorentzen S, Mjøsund $\mathrm{NH}$, Opjordsmoen S, et al. Characteristics of patients frequently subjected to pharmacological and mechanical restraint--a register study in three Norwegian acute psychiatric wards. Psychiatry Research. 2014;215(1):127-133. https://doi.org/10.1016/j.psychres.2013.10.024

39. Walsh JK. Pharmacologic management of insomnia. The Journal of Clinical Psychiatry. 2004;65 Suppl 16:41-45.

40. Chung $\mathrm{W}$, Cho WH, Yoon $\mathrm{CW}$. The influence of institutional characteristics on length of stay for psychiatric patients: A national database study in South Korea. Social Science \& Medicine. 2009;68(6):1137-1144. https://doi.org/10.1016/j.socscimed.2008.12.045

41. Clarke SP, Aiken LH. Failure to rescue: Needless deaths are prime examples of the need for more nurses at the bedside. The American Journal of Nursing. 2003;103(1):42-47. https://doi.org/10.1097/00000446-200301000-00020

42. Aiken LH, Clarke SP, Sloane DM. Hospital staffing, organiza- tion, and quality of care: Cross-national findings. International Journal for Quality in Health Care. 2002;14(1):5-13. https://doi.org/10.1093/intqhc/14.1.5

43. Cleary M, Horsfall J, O’Hara-Aarons M, Hunt GE. Mental health nurses' views of recovery within an acute setting. International Journal of Mental Health Nursing. 2013;22(3) :205212. https://doi.org/10.1111/j.1447-0349.2012.00867.x

44. Bodén R, Brandt L, Kieler H, Andersen M, Reutfors J. Early non-adherence to medication and other risk factors for rehospitalization in schizophrenia and schizoaffective disorder. Schizophrenia Research. 2011;133(1-3):36-41. https://doi.org/10.1016/j.schres.2011.08.024

45. You SJ, Chang HS, Kim MK, Choi YK, Sung YH, Kim ES, et al. Study of the utilization strategy of nursing personnel by types of medical institutions. Journal of Korean Clinical Nursing Research. 2007;13(1):157-172.

46. Cho E, Sloane DM, Kim EY, Kim S, Choi M, Yoo IY, et al. Effects of nurse staffing, work environments, and education on patient mortality: An observational study. International Journal of Nursing Studies. 2015;52(2):535-542. https://doi.org/10.1016/j.ijnurstu.2014.08.006

47. Laguna-Parras JM, Jerez-Rojas MR, García-Fernández FP, Carrasco-Rodríguez MD, Nogales-Vargas-Machuca I. Effectiveness of the 'sleep enhancement' nursing intervention in hospitalized mental health patients. Journal of Advanced Nursing. 2013;69(6):1279-1288. https://doi.org/10.1111/j.1365-2648.2012.06116.x

48. Butcher HK, Bulechek GM, Dochterman JMM, Wagner CM. Nursing interventions classification (NIC). 7th ed. St. Louis (MO) : Elsevier; 2018. p. 349.

49. Twigg DE, Myers H, Duffield C, Pugh JD, Gelder L, Roche $\mathrm{M}$. The impact of adding assistants in nursing to acute care hospital ward nurse staffing on adverse patient outcomes: An analysis of administrative health data. International Journal of Nursing Studies. 2016;63:189-200. https://doi.org/10.1016/j.ijnurstu.2016.09.008

50. Cho SJ. Impact of nurse staffing on inpatient health outcomes [dissertation]. Seoul: Seoul National University; 2015. p. $1-150$. 